apyrexia, earlier chest tube removal, earlier hospital discharge and better response to antibiotherapy. Thoracoscopy prior to thoracic drainage can be indicated as first line treatment of loculated empyema for children.

\section{INGUINAL HERNIA IN “GIRLS” RARELY REVEALS COMPLETE ANDROGEN INSENSITIVITY SYNDROME}

doi:10.1136/archdischild-2012-302724.1590

${ }^{1} \mathrm{~N}$ Kalfa, ${ }^{2} \mathrm{~F}$ Paris, ${ }^{1} \mathrm{~A}$ Andreica, ${ }^{2} \mathrm{~F}$ Audran, ${ }^{1} \mathrm{O}$ Maillet, ${ }^{2 \mathrm{P}}$ Philibert, ${ }^{3} \mathrm{RB}$ Galifer, ${ }^{4} \mathrm{C}$ Sultan. ${ }^{1}$ Pediatric Surgery; ${ }^{2}$ Pediatric Endocrinology; ${ }^{3} \mathrm{CHU}$ Montpellier, Montpellier; ${ }^{4} \mathrm{CHU}$ Montpellier, Paris, France

Background Aim According to literature, the incidence of complete androgen insensitivity syndrome (CAIS) revealed by inguinal hernia in "girls" is variable due to the clinical heterogeneity of the series. The aim of this study is to estimate the percentage of CAIS in children with female phenotype who presented with various forms of hernias.

Material and Methods This is a retrospective study based on a population of 129 "girls" treated for bilateral hernia repair. The gonads were assessed either by preoperative US or by intra operative direct examination. In case of CAIS suspicion, gonadic tissue was sampled, karyotyping and hormonal analysis were performed. Diagnosis of CAIS was confirmed by direct AR gene sequencing (exons 1-8).

Results We identified 2 cases of CAIS (mutations pS204N+delR615 and del F584). The percentage of CAIS depends on the population involved. On the entire series (including simple permeability of the peritoneo-vaginal channel, $n=129$ ), the rate of CAIS is low, $1.6 \%$. In case of clinical bilateral hernia whatever the content, digestive or gonadal, the rate of CAIS climbs at $6.9 \%$. For the bilateral gonadic hernias $(n=7)$, the rate of CAIS is $28.6 \%$.

Conclusions The incidence of CAIS among "girls" undergoing bilateral hernia repair is low and varies according to the involved population. The simple permeability of the contralateral channel is not a significant risk factor for CAIS. Systematic research of CAIS may be justified in a small number of patients, especially those with bilateral gonadal content. Visualization of the gonads remains mandatory in these particular patients.

\section{DETECTION OF HERPES VIRUSES IN CHILDREN WITH ACUTE APPENDICITIS}

doi:10.1136/archdischild-2012-302724.1591

'E Blevrakis, 'C Seremeti, ${ }^{2 P}$ Katzoli, ${ }^{2 M}$ Ergazaki, ${ }^{2} \mathrm{DA}$ Spandidos, ${ }^{2} \mathrm{G}$ Sourvinos, ${ }^{1} \mathrm{G}$ Sakellaris. 'Department of Paediatric Surgery, University Hospital of Heraklion, Crete; ¿Laboratory of Virology, Faculty of Medicine, University of Crete, Heraklion, Greece

Objective This study aimed to investigate the incidence of HSV types-1 and -2, VZV, CMV, EBV, HHV-6 and HHV-7 in childhood acute appendicitis.

Study Design Polymerase chain reaction (PCR) assays were applied to detect herpes virus DNA in 38 children [11 girls and 27 boys, mean age 9 years (STD \pm 2.59 ), range 6-14 years], who underwent an appendectomy within a 2.5 -year period. Appendix, omentum and peripheral blood mononuclear cells (PBMCs) were available from each case. Of the 38 children with acute appendicitis, $20(52.6 \%)$ had advanced (phlegmonous) acute appendicitis and $18(47.4 \%)$ had perforated appendicitis and local peritonitis. Fortyone blood specimens from age-matched healthy children (25 female and 16 male), with clinical manifestations unrelated to viral infections served as negative controls.

Results CMV was the most frequently detected virus (8/38.21\%), followed by HHV-6 (3/38.7.9\%). EBV and HSV-1 were detected, though not in all three different types of tissue specimens tested. None of the samples examined were HSV-2, VZV or HHV-7 positive.
Of all the specimens, the omentum was the most commonly infected tissue $(63.0 \%)$ while the appendix and peripheral blood specimens were found to be positive for viral infection in $60.5 \%$ and $50 \%$ of cases, respectively. The CMV IgG+ antibodies were positive in $54 \%$ of the control cases while $86 \%$ of the same group presented HHV-6 IgG+ antibodies.

Conclusion To the best of our knowledge, this is the first study documenting the presence of herpes virus DNA in children with acute appendicitis, suggesting that possible viral infection or reactivation is associated with childhood appendicitis.

\section{SACROCOCCYGEAL TERATOMA: 10 YEARS EXPERIENCE IN UPPER EGYPT}

doi:10.1136/archdischild-2012-302724.1592

'MA Osman, ${ }^{2} \mathrm{IA}$ Ibrahim. 'Pediatric Surgical Unit, Surgery Department; ${ }^{2}$ Pediatric Surgery Unit, Faculty of Medicine, Assiut University, Assiut, Egypt

Purpose To evaluate our experience of 45 patients with SCT (in Upper Egypt) over a period of 10 years (2001-2011), and to determine of the outcome of the management and recommendations for treatment's strategies.

Patients and Methods The records were reviewed for age at presentation, clinical manifestations and investigations, time of surgical approach, histopathology, recurrences, bladder and anorectal function, and cosmetic outcome.

Results The time of referral was immediately after birth in 5 patients, at the $1^{\text {st }}$ week in 17 , later in infancy in 22 , and at age of 1.5 years in one child. Excision of the lesion was done for 41 patients. Teratomas were type I $(n=9)$, type II $(n=20)$, type III $(n=11)$, type IV $(n=1)$ (Altman's classification). The age of operation ranged from 2 days to 1.5 years. Histology of results were: mature teratoma $(n=27)$, immature teratoma $(n=9)$, malignant teratoma $(n=5)$. Coccyx remained in two cases of early period of study.

FU ranged from 3 months to 10 years. Recurrence rate in 13 $(31.7 \%)$, wound infection occurred in $4(9.7 \%)$, diarrhea occurred in 2 (4.8\%). AFP was high in 35 and normal in two patients, it decreased after excision. Fetal diagnosis was made in 5 cases by prenatal sonography.

Conclusion Prenatal diagnosis of SCT is important and recommended to save the baby from obstructed labor. Early diagnosis allows early surgical intervention avoids malignant transformation. The coccyx should be excised to decrease the risk of recurrence. Skin flap modification is feasible for large teratomas with healthy skin.

\section{ANTENATALLY DIAGNOSED OVARIAN CYSTS: MANAGEMENT AND FOLLOW-UP PROTOCOL}

doi:10.1136/archdischild-2012-302724.1593

'F Laconi, 'V Guerriero, ${ }^{2} \mathrm{R}$ Raffaelli, ${ }^{3} \mathrm{M}$ Carli, ${ }^{4} \mathrm{FS}$ Camoglio. 'Pediatric Surgery; ${ }^{2}$ Ultrasound and Prenatal Diagnosis Service UO Obstetrics Gynecology OP, University of Verona, Policlinico G.B. Rossi; ${ }^{3}$ Ultrasound and Prenatal Diagnosis Service UO Obstetrics Gynecology OP, ${ }^{4}$ Pediatric Surgery, University of Verona, Policlincio G.B. Rossi, Verona, Italy

Objectives Abdominal cysts are frequently detected in fetuses through routine prenatal ultrasound.

In female fetuses such cysts are most often originated from ovaries under the stimulus of maternal hormones, and can grow to a rather large size

We present our survey of prenatally detected ovarian cysts (OC) and we propose a management and follow-up protocol.

Methods A retrospective review of all cases of abdominal cysts in female fetuses detected in our institution from jan. 2007 to jan. 2012 was conducted.

Among all cases of abdominal cystic formations, 28 were originated from the ovary, and resulted at antenatal US scan ranging in 